\title{
Conic type Caffarelli-Kohn-Nirenberg inequality on manifold with conical singularity
}

\author{
Ali Asghar Jafari ${ }^{1}$ - Mohsen Alimohammady ${ }^{1}$
}

Received: 29 October 2017 / Accepted: 26 February 2018 / Published online: 29 May 2018

(C) The Author(s) 2018

\begin{abstract}
In this paper, we consider a manifold with conical singularity and introduce weighted cone Sobolev spaces. We prove a conic type Caffarelli-Kohn-Nirenberg inequality and then apply this inequality to obtain a existence result of a nonlinear elliptic equation on weighted cone Sobolev space.
\end{abstract}

Keywords Caffarelli-Kohn-Nirenberg-type inequality · Nonlinear elliptic equations $\cdot$ Partial differential equations on manifolds

Mathematics Subject Classification 35D05 · 35J66 · 35R01

\section{Introduction}

Let $n \geq 3$ be an integer and let $a, b$ and $p$ be constants satisfying the following conditions

$$
-\infty<a<\frac{n-p}{p}, \quad a \leq b \leq a+1, \quad p \in(1, n), \quad q=\frac{n p}{n-p(1+a-b)} .
$$

Ali Asghar Jafari

aa.jafari@stu.umz.ac.ir

Mohsen Alimohammady

amohsen@umz.ac.ir

1 Department of Mathematics, Faculty of Mathematical Sciences, University of Mazandaran,

Babolsar 47416-1468, Iran 
Cafarelli, Kohn and Nirenberg [3], for all $u \in C_{0}^{\infty}\left(\mathbb{R}^{n}\right)$, have established that there exists a positive constant $C$ depending only on constants $a, b$ and $n$ such that the functional inequality

$$
\left(\int_{\mathbb{R}^{n}}|x|^{-b q}|u|^{q} d v\right)^{\frac{1}{q}} \leq C\left(\int_{\mathbb{R}^{n}}|x|^{-p a}|\nabla u|^{p} d v\right)^{\frac{1}{p}}
$$

holds, where $|x|$ is the Euclidean length of $x \in \mathbb{R}^{n}$, and $d v$ is the Euclidean volume element determined by the standard Euclidean metric. Note that the Caffarelli-KohnNirenberg inequality 1.2 contains the classical Sobolev inequality $(a=b=0)$ and the Hardy ( $a=0, b=1)$ as special cases, which have played important roles in many applications in Partial differential equations. Inequality 1.2 proves to be an important tool in studying degenerate elliptic problems. It is also related to the understanding of some important phenomena, such as best constants, extremal functions, and their qualitative properties $[9,12]$. Some of the directions that have been developed include the study of positive solutions to critical singular quasilinear elliptic problems related to the Caffarelli-Kohn-Nirenberg inequality [5,10,11]. Since most of the problems in differential geometry can be reduced to problems in differential equations on Riemannian manifolds, it is important to have in this case investigation tools similar to those successfully used in the Euclidean case. Many authors investigated some modified version of the inequality 1.2 to study some of degenerate elliptic system which contains differential operators of the type

$$
\operatorname{div}_{g}\left(w(x)\left|\nabla_{g} u\right|^{p}\right)
$$

where $w(x)$ is a non-negative function satisfying inf $w(x)=0$, on a Riemannian Manifold with Riemannain metric $g$, for example see $[1,2,4,16]$. Degenerate differential operators involving a non-negative weight which appear in singular quasilinear elliptic equations have been studied by some authors in Euclidean and Riemannian manifolds forms. In fact, the non-negative weight function $w(x)$ allow us to have zeros at some points or even to be unbounded are used in the study of many physical phenomena. In this article, we want to study a class of singular quasilinear differential equations on manifolds with conical singularities. Many applications of physics and applied sciences as well as of the structure mathematics lead to elliptic problems with singularities in the sense of non-smoothness of boundaries $[6,8]$.

\section{Conical manifolds and cone spaces}

In this section we recall some definitions and basic properties of manifolds with conical singularities and the corresponding cone Sobolev spaces. Then we will introduce a generalization of the cone Sobolev spaces on under suitable non-negative weight and the typical differential operators on those manifolds. For more details and proofs about the results on manifolds with conical singularities, we refer to Egorov and Schulze [7], Schulze [14], Schrohe and Seiler [15].

First we introduce some notations. 
(a) $\mathbb{R}_{+}=(0, \infty), \mathbb{R}_{+}^{-}=[0, \infty)$;

(b) $X$ is a smooth compact manifold without boundary;

(c) $X^{\wedge}:=\mathbb{R}_{+} \times X$, interpreted as the open stretched cone with base $X$;

(d) $X^{\Delta}:=\frac{\left(\mathbb{R}_{+} \times X\right)}{\{0\} \times X}$, interpreted as the cone.

Definition 2.1 Let $B$ be a Hausdorff topological compact space and $b \in B$ such that $B-\{b\}$ is a smooth manifold without boundary of dimension $n+1$. $B$ is called a manifold with conical singularity $b$ if the following properties hold:

(I) there exist a neighborhood $U$ of $b$ and a diffeomorphism

$$
\varphi: U-\{b\} \rightarrow X^{\wedge}
$$

for some closed compact smooth manifold $X$ without boundary of dimension $n$, (II) $\varphi$ is extendible to a homeomorphism $\bar{\varphi}: U \rightarrow X^{\Delta}$.

If one considers another diffeomorphism $\Phi: U-\{b\} \rightarrow X^{\wedge}$ extendible to $U$, we say that these two diffeomorphism are equivalent if $\varphi \Phi^{-1}: X^{\wedge} \rightarrow X^{\wedge}$ is the restriction of some diffeomorphism $\mathbb{R}_{+}^{-} \times X \rightarrow \mathbb{R}_{+}^{-} \times X$ to $\mathbb{R}_{+} \times X$. Therefore, we consider the map 2.1 fixed, then

$$
\delta_{\lambda} u:=\phi^{-1}(\lambda r, x), \phi(u)=(r, x), \lambda \in \mathbb{R}_{+}, u \in U-\{b\},
$$

induces an $\mathbb{R}_{+}$group action on $U-\{b\}$. The manifold $X$ is called the base of the cone and $\mathbb{R}_{+}$the cone axis. According to above assumptions and by described method in [7], there exists a smooth manifold $\mathbb{B}$ with compact $C^{\infty}$-boundary $\partial \mathbb{B} \equiv X$ for which there is a diffeomorphism $\mathbb{B}-\partial \mathbb{B} \equiv B-\{b\}$ the restriction of which $U_{1}-\{b\}$ is a diffeomorphism $U_{1}-\{b\} \equiv V_{1}-\partial \mathbb{B}$ for an open neighborhood $U_{1} \subset B$ of the conical point $b$ and a collar neighborhood $V_{1} \subset \mathbb{B}$ of $\partial \mathbb{B}$, i.e. $V_{1} \equiv[0,1) \times X$.

$\mathbb{B}$ is called the stretched manifold with conical singularity associated with $B$. Via this identification the analysis of differential operators on $B$ is carried out on $\mathbb{B}$. The underlying analysis of a manifold with a conic point, the corresponding differential operators are called cone-degenerate differential operators or Fuchs type operators which are given in local coordinates in a collar neighborhood $[0,1) \times X$ of the boundary by

$$
A=r^{-m} \sum_{k=0}^{m} a_{k}(r)\left(-r \frac{\partial}{\partial r}\right)^{k}
$$

where the coefficients $a_{k}(r) \in C^{\infty}\left([0,1), \operatorname{Diff}^{m-k}(X)\right), r^{-m}$ is also called a weight factor with the weight $-m$. The cone degeneracy shows up in the derivative $\left(-r \partial_{r}\right)$.

Definition 2.2 Let $B$ be a manifold with a conical singularity point $b$. Then $A \in$ $\operatorname{Diff}^{m}(B-\{b\})$ is said to be of Fuchs type if $b$ has a neighborhood such that $A$ in local coordinates $(r, x)$ is of Fuchs type in the sense of 2.3. By similarly way, one can say that $A \in \operatorname{Diff}^{m}\left(\int \mathbb{B}\right)$ is of Fuchs type if it is a neighborhood of $\partial \mathbb{B}$ of the form 2.3. 
Let $g_{X}(r)$ be a $r$-dependent family of Riemannian metric on a closed compact $C^{\infty}$. manifold $X$ which is infinitely differentiable in $r \in[0,1)$ and $g_{X}(0)$ depends only on $x$ and $d x$. Then

$$
g:=d r^{2}+r^{2} g_{X}(r)
$$

is a Riemannain metric on $X^{\wedge}$. The gradient and divergence operators with respect to this metric are first order operators, div $v_{g} \in \operatorname{Diff}^{1}\left(X^{\wedge}, T X^{\wedge}, \mathbb{R}\right), \nabla_{g} \in$ $\operatorname{Diff}^{1}\left(X^{\wedge}, \mathbb{R}, T X^{\wedge}\right)$, respectively. Here, $T X^{\wedge}$ is the cone tangent bundle which has a basis in local coordinates as follows:

$$
r \frac{\partial}{\partial r}, \frac{\partial}{\partial x_{1}}, \ldots, \frac{\partial}{\partial x_{n}} .
$$

This basis induces a dual basis in the cone cotangent bundle $T^{*} X^{\wedge}$ given by $\frac{d r}{r}, d x_{1}, \ldots, d x_{n}$. Now, without lose of generality, we consider $\mathbb{B}=[0,1) \times X$ that $X$ is an $(n-1)$-dimensional closed compact $C^{\infty}$-manifold which is regarded as the local model near the conical singularity points, and $\partial \mathbb{B}=\{0\} \times X$. Then the corresponding gradient operator with respect to the Riemannain metric 2.4 is denoted by $\nabla_{\mathbb{B}(g)}:=\left(x_{1} \partial_{x_{1}}, \partial_{x_{2}}, \ldots, \partial_{x_{n}}\right)$. Moreover, for a vector field $F$, we define the divergence operator on $\mathbb{B}$ with respect to Riemannain metric 2.4 as

$$
\operatorname{div}_{\mathbb{B}(g)}(F):=x_{1} \frac{\partial F}{\partial x_{1}}+\sum_{k=2}^{n} \frac{\partial F}{\partial x_{k}} .
$$

The operator $\Delta_{\mathbb{B}(g)}$ is defined by $\left(x_{1} \partial_{x_{1}}\right)^{2}+\partial_{x_{2}}^{2}+\cdots+\partial_{x_{n}}^{2}$, which is an elliptic operator with conical degeneration on the boundary $x_{1}=0$. The operator $\Delta_{\mathbb{B}(g)}$ is also called Fuchsian-Laplace operator. Near the boundary of stretched manifold $\mathbb{B}, \partial \mathbb{B}$, we will use coordinates $\left(x_{1}, x^{\prime}\right):=\left(x_{1}, x_{2}, \ldots, x_{n}\right)$ for $x_{1} \in[0,1)$ and $x^{\prime} \in X$. According to the Fuchsian-Laplace operator, gradient and divergence operators defined on manifold $\mathbb{B}$, for $2<p<\frac{n-2}{2}=2^{*}$, we introduce $A(p, \mathbb{B}, g):=\operatorname{div}_{\mathbb{B}}\left(\left|\nabla_{\mathbb{B}} u\right|^{p-2} \nabla_{\mathbb{B}} u\right)$ sa a cone-degenerate operator of second order acting between sections of trivial bundles $X^{\wedge}$ and $X^{\wedge}$. We call $A(p, \mathbb{B}, g)$ Fuchsian-P-Laplace operator. Note that the range of power $p$, to definition of Fuchsian-P-Laplace operator, can be considered rather than general, but we are interesting to the range $\left(2,2^{*}\right)$ for our boundary value problem that we will study about the existence results it in the last section.

Definition 2.3 For $x=\left(x_{1}, x^{\prime}\right) \in \mathbb{R}_{+} \times \mathbb{R}^{n-1}=\mathbb{R}_{+}^{n}$, we say that

$$
u(x) \in L_{p}\left(\mathbb{R}_{+}^{n}, \frac{d x_{1}}{x_{1}} d x^{\prime} ; w(x)\right):=L_{p, w}
$$

if

$$
\|u\|_{L_{p, w}}=\left(\int_{\mathbb{R}_{+}} \int_{\mathbb{R}^{n-1}} x_{1}^{n}|w(x) u(x)|^{p} \frac{d x_{1}}{x_{1}} d x^{\prime}\right)^{\frac{1}{p}}<\infty .
$$


Therefore, the weighted $L_{p, w}$-spaces with weight data $\gamma \in \mathbb{R}$ is denoted by

$$
L_{p}^{\gamma}\left(\mathbb{R}_{+}^{n}, \frac{d x_{1}}{x_{1}} d x^{\prime} ; w(x)\right):=L_{p, w}^{\gamma}
$$

and defined as follows: if $u\left(x_{1}, x^{\prime}\right) \in L_{p}^{\gamma}\left(\mathbb{R}_{+}^{n}, \frac{d x_{1}}{x_{1}} d x^{\prime} ; w(x)\right)$, then

$$
x_{1}^{-\gamma} u\left(x_{1}, x^{\prime}\right) \in L_{p}\left(\mathbb{R}_{+}^{n}, \frac{d x_{1}}{x_{1}} d x^{\prime} ; w(x)\right),
$$

and

$$
\|u\|_{L_{p, w}^{\gamma}}=\left(\int_{\mathbb{R}_{+}} \int_{\mathbb{R}^{n-1}} x_{1}^{n-\frac{\gamma}{p}}|w(x) u(x)|^{p} \frac{d x_{1}}{x_{1}} d x^{\prime}\right)^{\frac{1}{p}}<\infty .
$$

Now, we can define the weighted $p$-Sobolev spaces for $1 \leq p<\infty$.

Definition 2.4 For $m \in \mathbb{N}, \gamma \in \mathbb{R}$ and $1 \leq p<\infty$, the spaces

$$
\mathcal{H}_{p, w}^{m, \gamma}\left(\mathbb{R}_{+}^{n}\right):=\left\{u \in \mathcal{D}^{\prime}\left(\mathbb{R}_{+}^{n}\right) ; x_{1}^{\frac{n}{p}-\gamma}\left(x_{1} \partial_{x_{1}}\right)^{\alpha} \partial_{x^{\prime}}^{\beta} u \in L_{p}\left(\mathbb{R}_{+}^{n}, \frac{d x_{1}}{x_{1}} d x^{\prime} ; w(x)\right)\right\}
$$

for any $\alpha \in \mathbb{N}, \beta \in \mathbb{N}^{n-1}$ and $|\alpha|+|\beta| \leq m$. In the other words, if $u\left(x_{1}, x\right) \in$ $\mathcal{H}_{p, w}^{m, \gamma}\left(\mathbb{R}_{+}^{n}\right)$, then $\left(x_{1} \partial_{x_{1}}\right)^{\alpha} \partial_{x^{\prime}}^{\beta} u \in L_{p}^{\gamma}\left(\mathbb{R}_{+}^{n}, \frac{d x_{1}}{x_{1}} d x^{\prime} ; w(x)\right)$.

Hence, $\mathcal{H}_{p, w}^{m, \gamma}\left(\mathbb{R}_{+}^{n}\right)$ is a Banach space with norm

$$
\|u\|_{\mathcal{H}_{p, w}^{m, \gamma}\left(\mathbb{R}_{+}^{n}\right)}=\sum_{|\alpha|+|\beta| \leq m}\left(\int_{\mathbb{R}_{+}} \int_{\mathbb{R}^{n-1}} x_{1}^{n-\frac{\gamma}{p}}\left|w(x)\left(x_{1} \partial_{x_{1}}\right)^{\alpha} \partial_{x^{\prime}}^{\beta} u\left(x_{1}, x^{\prime}\right)\right|^{p} \frac{d x_{1}}{x_{1}} d x^{\prime}\right)^{\frac{1}{p}}
$$

Let $X$ be a closed compact $C^{\infty}$ manifold, and $\mathcal{U}=\left\{U_{1}, \ldots, U_{N}\right\}$ an open covering of $X$ by coordinate neighborhoods. If we fix a subordinate partition of unity $\left\{\varphi_{1}, \ldots, \varphi_{N}\right\}$ and charts $\chi_{j}: U_{j} \rightarrow \mathbb{R}^{n-1}, j=1, \ldots, N$. Then we say that $u \in \mathcal{H}_{p, w}^{m, \gamma}\left(X^{\wedge}\right)$ if and only if $u \in \mathcal{D}^{\prime}\left(X^{\wedge}\right)$ with the norm

$$
\|u\|_{\mathcal{H}_{p, w}^{m, \gamma}\left(X^{\wedge}\right)}=\left\{\sum_{j=1}^{N}\left\|\left(1 \times \chi_{j}^{*}\right)^{-1} \varphi_{j} u\right\|_{\mathcal{H}_{p, w}^{m, \gamma}\left(\mathbb{R}_{+}^{n}\right)}^{p}\right\}^{\frac{1}{p}}<\infty
$$

where $1 \times \chi_{j}^{*}: C_{0}^{\infty}\left(\mathbb{R}_{+} \times \mathbb{R}^{n-1}\right) \rightarrow C_{0}^{\infty}\left(\mathbb{R}_{+} \times U_{j}\right)$ is the pull-back function with respect to $1 \times \chi_{j}: \mathbb{R}_{+} \times U_{j} \rightarrow \mathbb{R}_{+} \times \mathbb{R}^{n-1}$. Denote the $\mathbf{H}_{p, w}^{m, \gamma}\left(X^{\wedge}\right)$ as subspace of $\mathcal{H}_{p, w}^{m, \gamma}\left(X^{\wedge}\right)$ which is defined as the closure of $C_{0}^{\infty}\left(X^{\wedge}\right)$ with respect to the norm $\|\cdot\|_{\mathcal{H}_{p, w}^{m, \gamma}\left(X^{\wedge}\right)}$. Now, we have the following definition: 
Definition 2.5 Let $\mathbb{B}=[0,1) \times X$ be the stretched manifold of the manifold $B$ with conical singularity. Then the cone Sobolev space $\mathcal{H}_{p, w}^{m, \gamma}(\mathbb{B})$, for $m \in \mathbb{N}, \gamma \in \mathbb{R}$ and $p \in(1, \infty)$, is defined by

$$
\mathcal{H}_{p, w}^{m, \gamma}(\mathbb{B})=\left\{u \in W_{l o c}^{m, p}(i n t \mathbb{B}) ; \kappa u \in \mathcal{H}_{p, w}^{m, \gamma}\left(X^{\wedge}\right)\right\}
$$

for any cut-off function $\kappa$ supported by a collar neighborhood of $[0,1) \times X$. Moreover, the subspace $\mathbf{H}_{p, w}^{m, \gamma}(\mathbb{B})$ of $\mathcal{H}_{p, w}^{m, \gamma}(\mathbb{B})$ is defined by

$$
\mathbf{H}_{p, w}^{m, \gamma}(\mathbb{B}):=[\kappa] \mathbf{H}_{p, w}^{m, \gamma}\left(X^{\wedge}\right)+[1-\kappa] W_{w, 0}^{m, p}(\operatorname{int} \mathbb{B}),
$$

where $W_{w, 0}^{m, p}($ int $\mathbb{B})$ denotes the closure of $C_{0}^{\infty}($ int $\mathbb{B})$ in weighted Sobolev space $W_{w}^{m, p}(\tilde{X})$ when $\tilde{X}$ is closed compact $C^{\infty}$ manifold of dimension $n$ containing $\mathbb{B}$ as a submanifold with boundary.

Definition 2.6 Let $\mathbb{B}=[0,1) \times X$. We say that $u(x) \in L_{p, w}^{\gamma}(\mathbb{B})$ with $1<p<$ $\infty, \gamma \in \mathbb{R}$, if

$$
\|u\|_{L_{p, w}^{\gamma}(\mathbb{B})}=\int_{\mathbb{B}} x_{1}^{n-\frac{\gamma}{p}}|w(x) u(x)|^{p}\left(\frac{d x_{1}}{x_{1}}\right) d x^{\prime}<\infty .
$$

\section{Nonlinear Dirichlet boundary weighted Fuchsian-p-Laplacian problems}

In this section, we first consider a weight function $w$ which is satisfying on some conditions and then we obtain a special case of the Caffarelli-Kohn-Nirenberg inequality on weighted cone Sobolev space. As an application of this inequality in the second part of this section, we consider non-linear Drichlet boundary weighted Fuchsian- $p$ Lapcian problem on manifold with conical singularity and obtain a existence result of the problem.

For each $x \in \mathbb{B}=[0,1) \times X$, we set $m_{i}=\inf _{x \in \mathbb{B}} x_{i}, M_{i}=\sup _{x \in \mathbb{B}} x_{i}$. For each $i \in\{1, \ldots, n\}$ let $w_{i}:\left[m_{i}, M_{i}\right] \rightarrow \mathbb{R}$ be a functions of class $C^{1}$. Let $\vec{w}: \mathbb{B} \rightarrow \mathbb{R}^{n}$ be defined by

$$
\vec{w}(x)=\left(w_{1}\left(x_{1}\right), w_{2}\left(x_{2}\right), \ldots, w_{n}\left(x_{n}\right)\right) .
$$

We assume that there exists $w_{0}>0$ a constant such that

$$
\operatorname{div}_{\mathbb{B}} \vec{w}(x) \geq w_{0}>0 \quad \forall x \in \mathbb{B} .
$$

Theorem 3.1 Suppose that $\vec{w}(x)$ is defined as above and satisfy conditions 3.1 Then there exists a positive constant $C$ such that

$$
\int_{\mathbb{B}}|u|^{p} \frac{d x_{1}}{x_{1}} d x^{\prime} \leq \int_{\mathbb{B}}|\vec{w}(x)|^{p}\left|\nabla_{\mathbb{B}} u(x)\right|^{p} \frac{d x_{1}}{x_{1}} d x^{\prime} \quad \forall u \in C_{0}^{1}(\mathbb{B}) .
$$


Proof

$$
\begin{aligned}
\operatorname{div}_{\mathbb{B}}\left(|u|^{p} \vec{w}(x)\right)= & x_{1} \partial_{x_{1}}\left(|u|^{p} w_{1}\left(x_{1}\right)\right)+\sum_{i=2}^{n} \partial_{x_{i}}\left(|u|^{p} w_{i}\left(x_{i}\right)\right) \\
= & x_{1}\left(p|u|^{p-2} u \partial_{x_{1}} u w_{1}\left(x_{1}\right)+|u|^{p} \partial_{x_{1}} w_{1}\left(x_{1}\right)\right) \\
& +\sum_{i=2}^{n}\left(p|u|^{p-2} u \partial_{x_{i}} u w_{i}\left(x_{i}\right)+|u|^{p} \partial_{x_{i}} w_{i}\left(x_{i}\right)\right) \\
= & p|u|^{p-2} u\left(x_{1} \partial_{x_{1}} u w_{1}\left(x_{1}\right)+\sum_{i=2}^{n} \partial_{x_{i}} u w_{i}\left(x_{i}\right)\right) \\
& +|u|^{p}\left(x_{1} \partial_{x_{1}} w_{1}\left(x_{1}\right)+\sum_{i=2}^{n} \partial_{x_{i}} w_{i}\left(x_{i}\right)\right) \\
= & |u|^{p} d i v_{\mathbb{B}} \vec{w}(x)+p|u|^{p-2} u \nabla_{\mathbb{B}} u . \vec{w}(x) .
\end{aligned}
$$

Moreover, one can conclude from the flux-convergence theorem that for each $u \in$ $C_{0}^{1}(\mathbb{B})$

$$
\int_{\mathbb{B}} \operatorname{div}_{\mathbb{B}}\left(|u|^{p} \vec{w}(x)\right) \frac{d x_{1}}{x_{1}} d x^{\prime}=\int_{\partial \mathbb{B}}|u|^{p} \vec{w}(x) \cdot \vec{n} d \sigma=0 .
$$

Therefore,

$$
\begin{aligned}
\int_{\mathbb{B}}|u|^{p} d i v_{\mathbb{B}} \vec{w}(x) \frac{d x_{1}}{x_{1}} d x^{\prime} & =-\int_{\mathbb{B}} p|u|^{p-2} u \nabla_{\mathbb{B}} u \cdot \vec{w}(x) \frac{d x_{1}}{x_{1}} d x^{\prime} \\
& \leq\left.\left|-\int_{\mathbb{B}} p\right| u\right|^{p-2} u \nabla_{\mathbb{B}} u \cdot \vec{w}(x) \frac{d x_{1}}{x_{1}} d x^{\prime} \mid \\
& \leq p \int_{\mathbb{B}}|u|^{p-1}\left|\nabla_{\mathbb{B}} u\right||\vec{w}(x)| \frac{d x_{1}}{x_{1}} d x^{\prime}
\end{aligned}
$$

A typical Young's inequality states that for any $\varepsilon>0$ and each $a, b \geq 0$ we have

$$
a b \leq \varepsilon a^{\frac{p}{p-1}}+\frac{1}{\varepsilon^{p-1}} b^{p} .
$$

Then we can take $\varepsilon>0$ such that $p \varepsilon<w_{0}$, where $w_{0}$ is given by relation3.1. Thus,

$$
\int_{\mathbb{B}}|u|^{p} \frac{d x_{1}}{x_{1}} d x^{\prime} \leq \int_{\mathbb{B}}|u|^{p} d i v_{\mathbb{B}} \vec{w}(x) \frac{d x_{1}}{x_{1}} d x^{\prime}
$$




$$
\begin{aligned}
& \leq p \int_{\mathbb{B}}|u|^{p-1}\left|\nabla_{\mathbb{B}} u\right||\vec{w}(x)| \frac{d x_{1}}{x_{1}} d x^{\prime} \\
& \leq p\left[\varepsilon \int_{\mathbb{B}}|u|^{p} \frac{d x_{1}}{x_{1}} d x^{\prime}+\int_{\mathbb{B}} \frac{1}{\varepsilon^{p-1}}|\vec{w}(x)|^{p}\left|\nabla_{\mathbb{B}} u\right|^{p} \frac{d x_{1}}{x_{1}} d x^{\prime}\right],
\end{aligned}
$$

On the other hand, for any $u \in C_{0}^{1}(\mathbb{B})$

$$
\left(w_{0}-\varepsilon p\right) \int_{\mathbb{B}}|u|^{p} \frac{d x_{1}}{x_{1}} d x^{\prime} \leq \frac{p}{\varepsilon^{p-1}} \int_{\mathbb{B}}|\vec{w}(x)|^{p}\left|\nabla_{\mathbb{B}} u\right|^{p} \frac{d x_{1}}{x_{1}} d x^{\prime} .
$$

Therefore

$$
\int_{\mathbb{B}}|u|^{p} \frac{d x_{1}}{x_{1}} d x^{\prime} \leq \frac{p}{\varepsilon^{p-1}\left(w_{0}-\varepsilon p\right)} \int_{\mathbb{B}}|\vec{w}(x)|^{p}\left|\nabla_{\mathbb{B}} u\right|^{p} \frac{d x_{1}}{x_{1}} d x^{\prime} .
$$

The proof of theorem is complete.

Remark 3.2 The result of Theorem 3.1 implies the fact that under condition 3.1 there exists a positive constant $K$ such that,

$$
\int_{\mathbb{B}}|u|^{p} \frac{d x_{1}}{x_{1}} d x^{\prime} \leq K \int_{\mathbb{B}}\left|\nabla_{\mathbb{B}} u\right|^{p} \frac{d x_{1}}{x_{1}} d x^{\prime} \forall u \in C_{0}^{1}(\mathbb{B}) .
$$

Hence, we deduce that

$$
\inf _{\mathbf{H}_{p, w}^{1, \frac{n}{p}}(\mathbb{B})-\{0\}} \frac{\int_{\mathbb{B}}\left|\nabla_{\mathbb{B}} u\right|^{p} \frac{d x_{1}}{x_{1}} d x^{\prime}}{\int_{\mathbb{B}}|u|^{p} \frac{d x_{1}}{x_{1}} d x^{\prime}}>0 .
$$

The above relation asserts that in this case the first eigenvalue of the Fuchsian-pLaplacian operator.

Now, we show the existence of weak solution for problem

$$
\begin{cases}-\operatorname{div}_{\mathbb{B}}\left(|w(x)|^{p}\left|\nabla_{\mathbb{B}} u\right|^{p-2} \nabla_{\mathbb{B}} u\right)+u=\lambda|u|^{q-2} u & x \in i n t \mathbb{B}, \\ u=0 & x \in \partial \mathbb{B}\end{cases}
$$

where $2<q<p<2^{*}$ and $\lambda$ is a positive constant. We say that $u \in \mathbf{H}_{p, w}^{1, \frac{n}{p}}(\mathbb{B})$ is a weak solution of problem 3.9 if

$$
\int_{\mathbb{B}}|\vec{w}(x)|^{p}\left|\nabla_{\mathbb{B}} u\right|^{p-2} \nabla_{\mathbb{B}} u \nabla_{\mathbb{B}} v \frac{d x_{1}}{x_{1}} d x^{\prime}+\int_{\mathbb{B}} u v \frac{d x_{1}}{x_{1}} d x^{\prime}-\lambda \int_{\mathbb{B}}|u|^{q-2} u v \frac{d x_{1}}{x_{1}} d x^{\prime}=0
$$

for any $v \in \mathbf{H}_{p, w}^{1, \frac{n}{p}}(\mathbb{B})$. 
The following proposition will be used for solving our problem.

Proposition 3.3 If $2 \leq q<p<2^{*}$ then $\mathbf{H}_{p, w}^{1, \frac{n}{p}}(\mathbb{B}) \hookrightarrow \mathcal{H}_{q, 0}^{0, \frac{n}{q}}(\mathbb{B})$ is compact.

Proof From Definition 2.5

$$
\mathbf{H}_{p, w}^{1, \frac{n}{p}}(\mathbb{B})=[\kappa] \mathcal{H}_{p, 0}^{1, \frac{n}{p}, a}(\hat{\mathrm{X}})+[1-\kappa] \mathrm{W}_{w, 0}^{1, p}(\text { int } \mathbb{B})
$$

and

$$
\mathcal{H}_{q, 0}^{0, \frac{n}{q}}(\mathbb{B})=[\kappa] \mathcal{H}_{q, 0}^{0, \frac{n}{q}}(\hat{\mathrm{X}})+[1-\kappa] L^{q}(\text { int } \mathbb{B}) .
$$

From Theorem 3.1 and classic embedding theorem we have the embedding [1 $\kappa] \mathrm{W}_{w, 0}^{1, p}($ int $\mathbb{B}) \hookrightarrow[1-\kappa] L^{q}($ int $\mathbb{B})$ is compact for $2 \leq q<p<2^{*}$. In fact, let $u_{n}$ be bounded sequence in $\mathrm{W}_{w, 0}^{1, p}(i n t \mathbb{B})$. By Theorem 1 it follows that $u_{n}$ is a bounded sequence in $L^{p}($ int $\mathbb{B})$. Consequently, $u_{n} \subset \mathrm{W}_{0}^{1, p}($ int $\mathbb{B})$ is a bounded sequence. The classical compact embedding theorem shows that there exists a convergence subsequence of $u_{n}$, still denoted by $u_{n}$, in $L^{q}($ int $\mathbb{B})$. Thus $[1-\kappa] \mathrm{W}_{w, 0}^{1, p}($ int $\mathbb{B})$ is compactly embedded in $L^{q}(i n t \mathbb{B})$. It is sufficient to show that the embedding $[\kappa] \mathcal{H}_{p, w}^{1, \frac{n}{p}}(\hat{\mathrm{X}}) \hookrightarrow[\kappa] \mathcal{H}_{q, 0}^{0, \frac{n}{q}}(\hat{\mathrm{X}})$ is compact. For $m \in \mathbb{N}, \gamma \in \mathbb{R}$, and $1<l<\infty$, we define

$$
\left(S_{\frac{n}{l}, \gamma} v\right)\left(r, x^{\prime}\right)=e^{-r\left(\frac{n}{l}-\gamma\right)} v\left(e^{-r}, x^{\prime}\right)
$$

for $v\left(x_{1}, x^{\prime}\right) \in \mathcal{H}_{l, w}^{m, \gamma}(\hat{\mathrm{X}})$. Then $S_{\frac{n}{l}, \gamma}$ induces an isomorphism as follows,

$$
S_{\frac{n}{l}, \gamma}:[\kappa] \mathcal{H}_{l, w}^{m, \gamma}(\hat{\mathrm{X}}) \rightarrow[\tilde{\kappa}] \mathrm{W}_{w}^{m, l}(\mathbb{R} \times \mathrm{X}),
$$

with a cut-off function $\tilde{\kappa}(r)=\kappa\left(e^{-r}\right) \in C_{0}^{\infty}\left(\mathbb{R}_{+}\right)$. In the present case, we take $\gamma=\frac{n}{q}$, and $l=q$ for $u\left(x_{1}, x^{\prime}\right) \in \mathcal{H}_{q}^{0, \frac{n}{q}}(\hat{\mathrm{X}})$. Thus we have the following isomorphism mapping

$$
S_{\frac{n}{q}, \frac{n}{q}}:[\kappa] \mathcal{H}_{q}^{0, \frac{n}{q}}(\hat{\mathrm{X}}) \rightarrow[\tilde{\kappa}] \mathrm{L}^{q}(\mathbb{R} \times \mathrm{X}),
$$

i.e.

$$
S_{\frac{n}{q}, \frac{n}{q}}\left(\kappa\left(x_{1}\right) u\left(x_{1}, x^{\prime}\right)\right)=\kappa\left(e^{-r}\right) e^{-r\left(\frac{n}{q}-\frac{n}{q}\right)} u\left(e^{-r}, x^{\prime}\right)=\kappa\left(e^{-r}\right) u\left(e^{-r}, x^{\prime}\right) .
$$

Analogously if we take $l=p, \gamma=\frac{n}{p}$ and $v\left(x_{1}, x^{\prime}\right) \in \mathcal{H}_{p, w}^{1, \frac{n}{p}}(\hat{\mathrm{X}})$, then we have an isomorphism

$$
S_{\frac{n}{p}, \frac{n}{p}}:[\kappa] \mathcal{H}_{p, w}^{1, \frac{n}{p}}(\hat{\mathrm{X}}) \rightarrow[\tilde{\kappa}] \mathrm{W}_{w, 0}^{1, p}(\mathbb{R} \times \mathrm{X})
$$


i.e.

$$
S_{\frac{n}{p}, \frac{n}{p}}\left(\kappa\left(x_{1}\right) v\left(x_{1}, x^{\prime}\right)\right)=\kappa\left(e^{-r}\right) e^{-r\left(\frac{n}{p}-\frac{n}{p}\right)} v\left(e^{-r}, x^{\prime}\right)=\kappa\left(e^{-r}\right) v\left(e^{-r}, x^{\prime}\right) .
$$

Moreover, $S_{\frac{n}{q}, \frac{n}{q}}$ induces another isomorphism. In fact, for every $v\left(x_{1}, x^{\prime}\right) \in \mathcal{H}_{p, w}^{1, \frac{n}{p}}(\hat{\mathrm{X}})$, one has

$$
\begin{aligned}
S_{\frac{n}{q}, \frac{n}{q}}\left(\kappa\left(x_{1}\right) v\left(x_{1}, x^{\prime}\right)\right) & =\kappa\left(e^{-r}\right) e^{-r\left(\frac{n}{q}-\frac{n}{q}\right)} v\left(e^{-r}, x^{\prime}\right) \\
& =\kappa\left(e^{-r}\right) e^{-r\left(\frac{n}{p}-\frac{n}{p}\right)} e^{r\left(\frac{n}{p}-\frac{n}{p}\right)} e^{-r\left(\frac{n}{q}-\frac{n}{q}\right)} v\left(e^{-r}, x^{\prime}\right) \\
& =\kappa\left(e^{-r}\right) e^{-r\left(\frac{n}{p}-\frac{n}{p}\right)} v\left(e^{-r}, x^{\prime}\right) \\
& =S_{\frac{n}{p}, \frac{n}{p}}\left(\kappa\left(x_{1}\right) v\left(x_{1}, x^{\prime}\right)\right) .
\end{aligned}
$$

and then the mapping

$$
S_{\frac{n}{q}, \frac{n}{q}}:[\kappa] \mathcal{H}_{p, w}^{1, \frac{n}{p}}(\hat{\mathrm{X}}) \rightarrow[\tilde{\kappa}] \mathrm{W}_{w, 0}^{1, p}(\mathbb{R} \times \mathrm{X})
$$

is also an isomorphism. Since the embedding $[\tilde{\kappa}] \mathrm{W}_{w, 0}^{1, p}(\mathbb{R} \times \mathrm{X}) \hookrightarrow[\tilde{\kappa}] \mathrm{L}^{q}(\mathbb{R} \times \mathrm{X})$ is compact, so from isomorphisms 3.10 and 3.11 the embedding $[\kappa] \mathcal{H}_{p, w}^{1, \frac{n}{p}}(\hat{\mathrm{X}}) \hookrightarrow$ $[\kappa] \mathcal{H}_{q, 0}^{0, \frac{n}{q}}(\hat{\mathrm{X}})$ is compact.

Theorem 3.4 The Dirichlet boundary value problem 3.9 has a non-trivial solution in the weighted Sobolev space $\mathbf{H}_{p, w}^{1, \frac{n}{p}}(\mathbb{B})$.

Proof In order to prove Theorem 3.4, we define,for each $\lambda>0$, the energy functional associated with problem 3.9 , which is defined on $\mathbf{H}_{p, w}^{1, \frac{n}{p}}(\mathbb{B})$,

$$
\begin{aligned}
J_{\lambda}(u)= & \frac{1}{p} \int_{\mathbb{B}}|\vec{w}(x)|^{p}\left|\nabla_{\mathbb{B}} u\right|^{p} \frac{d x_{1}}{x_{1}} d x^{\prime}+\frac{1}{2} \int_{\mathbb{B}}|u|^{2} \frac{d x_{1}}{x_{1}} d x^{\prime} \\
& -\frac{\lambda}{q} \int_{\mathbb{B}}|u|^{q} \frac{d x_{1}}{x_{1}} d x^{\prime} \quad \forall u \in \mathbf{H}_{p, w}^{1, \frac{n}{p}}(\mathbb{B}) .
\end{aligned}
$$

Standard arguments show that $J_{\lambda} \in C^{1}\left(\mathbf{H}_{p}^{1, \frac{n}{p}}(\mathbb{B}), \mathbb{R}\right)$ and its derivative is given by

$$
\begin{aligned}
\left\langle J_{\lambda}^{\prime}(u), v\right\rangle= & \int_{\mathbb{B}}|\vec{w}(x)|^{p}\left|\nabla_{\mathbb{B}} u\right|^{p-2} \nabla_{\mathbb{B}} u \nabla_{\mathbb{B}} v \frac{d x_{1}}{x_{1}} d x^{\prime}+\int_{\mathbb{B}} u v \frac{d x_{1}}{x_{1}} d x^{\prime} \\
& -\lambda \int_{\mathbb{B}}|u|^{q-2} u v \frac{d x_{1}}{x_{1}} d x^{\prime},
\end{aligned}
$$


for all $u, v \in \mathbf{H}_{p, w}^{1, \frac{n}{p}}(\mathbb{B})$. We infer that $u$ is a solution of problem 3.9 if and only if it is a critical point of $J_{\lambda}$. Consequently, we concentrate our efforts on finding critical points of $J_{\lambda}$. In this context we prove the following assertion:

(a) The functional $J_{\lambda}$ is weakly lower semi-continuous.

(b) The functional $J_{\lambda}$ is bounded from below and coercive.

(c) There exists $\psi \in \mathbf{H}_{p, w}^{1, \frac{n}{p}}(\mathbb{B})-\{0\}$ such that $J_{\lambda}(\psi)<0$.

The arguments as in the prove $(a),(b)$ and $(c)$ are detailed below.

(a) By the Poincare' inequality, there exist constant $C$, such that the following estimates

$$
\|u\|_{L_{p, w}^{\frac{n}{p}}(\mathbb{B})} \leq\|u\|_{\mathbf{H}_{p, w}^{1, \frac{n}{p}}(\mathbb{B})} \leq C\left\|\nabla_{\mathbb{B}} u\right\|_{L_{p, w}^{\frac{n}{p}}(\mathbb{B})}
$$

hold, so we will use the standard form $\|u\|_{\mathbf{H}_{p, w}^{1, \frac{n}{p}}(\mathbb{B})}=\left\|\nabla_{\mathbb{B}} u\right\|_{L_{p, w}^{\frac{n}{p}}(\mathbb{B})}$.

$$
\begin{aligned}
J_{\lambda}(u)= & \frac{1}{p} \int_{\mathbb{B}}|\vec{w}(x)|^{p}\left|\nabla_{\mathbb{B}} u\right|^{p} \frac{d x_{1}}{x_{1}} d x^{\prime}+\frac{1}{2} \int_{\mathbb{B}}|u|^{2} \frac{d x_{1}}{x_{1}} d x^{\prime} \\
& -\frac{\lambda}{q} \int_{\mathbb{B}}|u|^{q} \frac{d x_{1}}{x_{1}} d x^{\prime} \quad \forall u \in \mathbf{H}_{p, w}^{1, \frac{n}{p}}(\mathbb{B}),
\end{aligned}
$$

Suppose

$$
I(u)=\frac{1}{p} \int_{\mathbb{B}}|\vec{w}(x)|^{p}\left|\nabla_{\mathbb{B}} u\right|^{p} \frac{d x_{1}}{x_{1}} d x^{\prime}+\frac{1}{2} \int_{\mathbb{B}}|u|^{2} \frac{d x_{1}}{x_{1}} d x^{\prime},
$$

by Sobolev embedding there exist a constant $C_{1}$ such that

$$
I(u) \leq \frac{1}{p} \int_{\mathbb{B}}|\vec{w}(x)|^{p}\left|\nabla_{\mathbb{B}} u\right|^{p} \frac{d x_{1}}{x_{1}} d x^{\prime}+\frac{C_{1}}{2} \int_{\mathbb{B}}|u|^{p} \frac{d x_{1}}{x_{1}} d x^{\prime} .
$$

Now apply Theorem 3.1 there exist a constant $C_{2}$ such that

$$
I(u) \leq \frac{1}{p} \int_{\mathbb{B}}|\vec{w}(x)|^{p}\left|\nabla_{\mathbb{B}} u\right|^{p} \frac{d x_{1}}{x_{1}} d x^{\prime}+\frac{C_{1} C_{2}}{2} \int_{\mathbb{B}}|\vec{w}(x)|^{p}\left|\nabla_{\mathbb{B}} u\right|^{p} \frac{d x_{1}}{x_{1}} d x^{\prime},
$$

accordingly there exist a constant $C$ which $I(u) \leq C\left\|\nabla_{\mathbb{B}} u\right\|_{L_{p, w}^{p}(\mathbb{B})}^{p}$. So $I(u)$ is weakly lower semi-continuous. That is, let $\left(u_{n}\right) \subset \mathcal{H}_{p, w}^{1, \frac{n}{p}}(\mathbb{B})$ be a sequence which weakly convergence to $u$ in $\mathbf{H}_{p, w}^{1, \frac{n}{p}}(\mathbb{B})$, Then we have

$$
I(u) \leq \liminf _{n \rightarrow \infty} I\left(u_{n}\right)
$$


On the other hand, since $\mathbf{H}_{p, w}^{1, \frac{n}{p}}(\mathbb{B})$ is compactly embedded in $L_{q}^{\frac{n}{q}}(\mathbb{B})$ it follows that $\left(u_{n}\right)$ convergence strongly to $\mathrm{u}$ in $L_{q}^{\frac{n}{q}}(\mathbb{B})$ that is,

$$
\frac{\lambda}{q} \int_{\mathbb{B}}|u|^{q} \frac{d x_{1}}{x_{1}} d x^{\prime}=\liminf _{n \rightarrow \infty} \frac{\lambda}{q} \int_{\mathbb{B}}\left|u_{n}\right|^{q} \frac{d x_{1}}{x_{1}} d x^{\prime}
$$

such that $J_{\lambda}(u) \leq \liminf _{n \rightarrow \infty} J_{\lambda}\left(u_{n}\right)$. Therefore, $J_{\lambda}$ is weakly lower semicontinuous.

(b) By proposition 3.3 for any $u \in \mathbf{H}_{p, w}^{1, \frac{n}{p}}(\mathbb{B})$, there exists a positive constant $K$ such that

$$
J_{\lambda}(u) \geq \frac{1}{p}\|u\|_{\mathbf{H}_{p, w}^{1, \frac{n}{p}}(\mathbb{B})}^{p}-\frac{K \lambda}{q}\|u\|_{\mathbf{H}_{p, w}^{1, \frac{n}{p}(\mathbb{B})}}^{q} .
$$

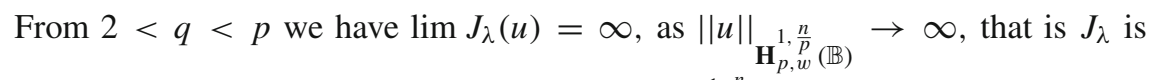
coercive. On the other hand, it is clear that for $u \in \mathbf{H}_{p, w}^{1, \frac{n}{p}}(\mathbb{B})$ we have

$$
J_{\lambda}(u) \geq\left(\frac{1}{p}\|u\|_{\mathbf{H}_{p, w}^{1, \frac{n}{p}}(\mathbb{B})}^{p-q}-\frac{K \lambda}{q}\right) \underset{\mathbf{H}_{p, w}^{1, \frac{n}{p}}(\mathbb{B})}{\|u\|^{q}}
$$

and thus $J_{\lambda}$ is bounded from below.

(c) We fix $0 \neq \varphi \in C_{c}^{1}(\mathbb{B})$, Then for each $t \in(0,1)$

$$
J_{\lambda}(t \varphi)=\frac{1}{p} \int_{\mathbb{B}}|\vec{w}(x)|^{p}\left|\nabla_{\mathbb{B}} t \varphi\right|^{p} \frac{d x_{1}}{x_{1}} d x^{\prime}+\frac{1}{2} \int_{\mathbb{B}}|t \varphi|^{2} \frac{d x_{1}}{x_{1}} d x^{\prime}-\frac{\lambda}{q} \int_{\mathbb{B}}|t \varphi|^{q} \frac{d x_{1}}{x_{1}} d x^{\prime} .
$$

Applying Sobolev embedding and Theorem 3.1 there exist $C$ such that

$$
J_{\lambda}(t \varphi) \leq C t^{p} \int_{\mathbb{B}}|\vec{w}(x)|^{p}\left|\nabla_{\mathbb{B}} \varphi\right|^{p} \frac{d x_{1}}{x_{1}} d x^{\prime}-\frac{\lambda t^{q}}{q} \int_{\mathbb{B}}|\varphi|^{q} \frac{d x_{1}}{x_{1}} d x^{\prime}
$$

that $q<p$.

Thus, there exist two positive constants $L_{1}$ and $L_{2}$ such that for each $t \in(0,1)$

$$
J_{\lambda}(t \varphi) \leq L_{1} t^{p}-L_{2} t^{q}
$$

By this inequality, we infer that for any $t \in\left(0, \min \left\{1,\left(\frac{L_{2}}{L_{1}}\right)^{\frac{1}{p-q}}\right\}\right)$

$$
J_{\lambda}(t \varphi)<0
$$

From $(a),(b)$ and $(c)$ in relation to [13] theorem 1.2 shows that there exists $u_{\lambda} \in$ $\mathbf{H}_{p, w}^{1, \frac{n}{p}}(\mathbb{B})$ as a global minimum point of $J_{\lambda}$. Moreover, from $(c)$ we have $u_{\lambda} \neq 0$. 
Standard arguments based on theorem(2) we have that $u_{\lambda}$ is a critical point of $J_{\lambda}$ and thus, a nontrivial weak solution of problem 3.9, so the proof of Theorem 3.4 will be complete.

Open Access This article is distributed under the terms of the Creative Commons Attribution 4.0 International License (http://creativecommons.org/licenses/by/4.0/), which permits unrestricted use, distribution, and reproduction in any medium, provided you give appropriate credit to the original author(s) and the source, provide a link to the Creative Commons license, and indicate if changes were made.

\section{References}

1. Alimohammady, M., Kalleji, M.K.: Existence result for a class of semilinear totally characteristic hypoelliptic equations with conical degeneration. J. Funct. Anal. 265, 2331-2356 (2013)

2. Bozhkov, Y.: A Caffarelli-Kohn-Nirenberg type inequality on Riemannian manifolds. Appl. Math. Lett. 23, 1166-1169 (2010)

3. Caffarelli, L., Kohn, R., Nirenberg, L.: First order interpolation inequalities with weights. Compos. Math. 53, 259-275 (1984)

4. Chen, H., Liu, X., Wei, Y.: Existence theory for a class of semilinear totally characteristic elliptic equations with critical cone Sobolev exponents. Ann. Glob. Anal. Geom. 37, 27-43 (2011)

5. Costa, D.G.: Some new and short proofs for a class of Caffarelli-Kohn-Nirenberg type inequalities. J. Math. Anal. Appl. 337, 311-317 (2008)

6. D ávila, J., Perla, I.: Nonlinear elliptic problems with a singular weight on the boundary. Calc. Var. Partial Differ. Equ. 41(3-4), 567-586 (2011)

7. Egorov, V., Schulze, B.W.: Pseudo-Differential Operators, Singularities, Applications. Birkhäuser Verlage, Basel (1997)

8. Ghoussoub, N., Robert, F.: Sobolev inequalities for the Hardy-Schrdinger operator: extremals and critical dimensions. Bull. Math. Sci. 6(1), 89-144 (2016)

9. Hardy, G.H., Littlewood, J.E., Plya, G.: Inequalities, 2nd edn. University Press, Cambridge (1952)

10. Kang, D.: Positive solutions to the wehted critical quasilinear problems. Appl. Math. Comput. 213, 432-439 (2009)

11. Kang, D.: Some properties of solutions to the singular quasilinear problems. Nonlinear Anal. 72, 682-688 (2010)

12. Pucci, P., Servadei, R.: Existence, non-existence and regularity of radial ground states for p-Laplacain equations with singular weights. Ann. Inst. H. Poincare Anal. Non Lineaire 25, 505-537 (2008)

13. Struwe, M.: Variational Methods: Applications to Nonlinear Partial Differential Equations and Hamiltonian Systems. Springer, Heidelberg (1996)

14. Schulze, B.W.: Boundary Value Problems and Singular Pseudo-Differential Operators. Wiley, Chichester (1998)

15. Schrohe, E., Seiler, J.: Ellipticity and invertibility in the cone algebra on $L_{p}$-Sobolev spaces. Integr. Equ. Oper. Theory 41, 93-114 (2001)

16. Xia, C.: The Caffarelli-Kohn-Nirenberg inequalities on complete manifolds. Math. Res. Lett. 14, 875-885 (2007) 